\title{
Effect of sarcopenia on postoperative complications after curative pancreatectomy for adenocarcinoma
}

\author{
Ümit Mercan, Ogün Erşen, Ali Ekrem Ünal*
}

Surgical Oncology, General Surgery Department, Faculty of Medicine, Ankara University, Ankara, Turkey.

*Corresponding Author: Ümit Mercan (E-mail: umit.mercan@yahoo.com.tr)

(Submitted: 25 February 2020 - Revised version received: 09 March 2020 - Accepted: 26 March 2020 - Published online: 26 April 2020)

\begin{abstract}
Objective Despite the increased experience in pancreatic cancer surgery, morbidity and mortality rates remain very high. Many factors play a role in the development of postoperative complications and in studies it has been shown that sarcopenia, which is defined as progressive muscle mass loss is also an important factor. Despite the proven effect of sarcopenia on postoperative complications and oncological outcomes in many types of gastrointestinal cancer, there are very few studies on pancreatic cancer. Therefore, in this study, we aimed to investigate the effect of sarcopenia on the development of serious postoperative complications in patients who underwent curative pancreatectomy for pancreatic cancer

Methods Total psoas index (TPI) was calculated for sarcopenia diagnosis by measures of psoas muscle area on the level of L3 spine from preoperative staging computed tomograhpy $(\mathrm{CT})$ images in pancreatic cancer patients undergoing curative resection. Patient demographics and postoperative outcomes were analyzed in sarcopenic and non-sarcopenic group.

Results: It has been found to be statistically significant relation between severe postoperative complication and sarcopenia $(p=0,001>$ ) Sarcopenia was more associated with cardiac and pulmonary complications among others ( $p=0.007,0.003$ respectively). In multivariate analysis, age [odds ratio (OR): 1,08. 95\% confidence interval (Cl): 1,01 1,15, $p=0,013$ ], ASA score (OR: 2,84. 95\% Cl 1,62 4,97. $p=0,043)$ and TPI (OR: 3,61. 95\% Cl: 1,58 5,74. $p=0,001>$ ) has been found independent risk factors for severe postoperative complications.

Conclusion Our results suggest that sarcopenia determined by using TPI, which can be easily obtained by examining the preoperative CT imaging, is an independent risk factor of severe postoperative complications. Determining the degree of sarcopenia can affect patient selection, predictability of possible serious complications, elective operation preparation process with a combination of nutrition and exercise therapy in a particular patient group, and decisions regarding adjuvant or neoadjuvant therapy.

Key Words Pancreatectomy, Postoperative complication, Sarcopenia
\end{abstract}

\section{Introduction}

Although pancreatic adenocarcinoma constitutes a low percentage of gastrointestinal malignancies, it is still a type of cancer with a high mortality and 5-year survival rates that cannot exceed $1 \%$, since it is usually diagnosed at an advanced stage and few of the diagnosed patients have a chance of curative surgery. ${ }^{1,2}$ Despite numerous successes in medical treatments in the historical process, surgical resection is the basic step of pancreatic cancer treatment. ${ }^{3}$ Although surgical treatment can cure patients, despite the increased experience, the postoperative serious complication and morbidity rates are quite high. ${ }^{4}$ Recognizing the postoperative complications and ensuring that patients are delivered to adjuvant treatment as soon as possible by applying the necessary treatments are perhaps as important as the success of surgical resection. ${ }^{5,6}$ Moreover, these complications should not only be recognized and treated appropriately, but also various methods should be developed to predict the complications. Thus, the surgeon will be able to better identify the patient at high risk for postoperative complications. ${ }^{7,8}$

In recent studies, it has been found that sarcopenia - considered as a syndrome characterized by progressive loss of skeletal muscle mass - is one of many factors that increase the risk of serious postoperative complications especially in elderly patients. ${ }^{9-12}$ In addition to anthropometric measurements, the diagnosis of sarcopenia can be made by measurement of the cross-sectional images of psoas muscle in abdominal computed tomography (CT). ${ }^{13}$

In addition to the effect of sarcopenia on postoperative results, it has been shown in many studies that it affects oncologic outcomes in other gastrointestinal cancers such as colorectal cancer and gastric cancer. ${ }^{14-16}$ However, there are limited studies on the relationship between postoperative outcomes and sarcopenia in patients with pancreatic cancer. Therefore, in this study, we aimed to investigate the effect of sarcopenia on severe postoperative complications in patients undergoing curative resection for pancreatic adenocancer.

\section{Material and Methods}

\section{Patient Selection and Data Collection}

Between January 2014 and January 2020, 120 patients who underwent curative resection for pancreatic cancer at Ankara University Surgical Oncology Clinic were evaluated retrospectively. Demographic data, laboratory results, preoperative CT images, surgical and pathology reports, medical follow-up records were obtained through the hospital database. Patients with distant metastasis, who were evaluated as intraoperative unresectable, who underwent palliative operations, whose data could not be accessed and whose CT scans could not be measured appropriately or CT images were not found excluded from the study. Pancreaticoduodenectomy (PD), distal pancreatectomy (DP) or total pancreatectomy (TP) were performed when appropriate and the patients were divided into two groups as PD and non-PD. Pathological stages of patients were determined according to Japan Pancreas Society (2009) General rules for the study of pancreatic cancer, 6th edn. ${ }^{17}$ Postoperative complications were categorized and graded acoording to the Modified Clavien Dindo Classification. ${ }^{18}$ Pancreatic fistulas were categorized using the International Pancreatic Fistula Study definitions. ${ }^{19}$. 


\section{Computed Tomography Scanning Measurements}

Preoperative contrast-enhanced CT scans were analyzed to create a sarcopenia profile for each patient. The mean values of all measurements were taken as a basis. Cross-sectional area of both psoas muscles were obtained by manually drawing the outer edges of the psoas muscles on the lumbar (L) 3 vertebra image where both spinous proceses were observed.

\section{Sarcopenia Quantification}

Patients in the lowest $25 \%$ of the values obtained as a result of the total posoas index (TPI) determined specifically for gender were evaluated sarcopenically. Measurement of the psoas muscle area at the L3 lumbar vertebra level and the calculation of the patient's height and TPI have been described in other studies. ${ }^{9}$ If formulated, TPI is calculated by dividing the right and left psoas muscle by the total area of the individual in square meters of height [(right psoas muscle area + left psoas muscle area) / height $\left.\left(\mathrm{m}^{2}\right)\right]$.

\section{Statistical Analysis}

Numerical data are given as mean \pm standard deviation. Student's $t$-test, Mann-Withney $U$ test, Chi-square test or Fisher Exact test were used when appropriate for numerical and categorical variables. Binary logistic regression analysis was applied for possible factors affecting postoperative complications. $p$-Values below 0,05 were considered statistically significant. IBM Statistics version 23.0 was used for statistical analysis.

\section{Results}

There were 120 patients who met the study and analysis criteria. The relationship between clinicopathological variables and sarcopenia have been summarized in Table 1 . The mean age of the patients was $58,03 \pm 7,61$, of which $76(63,3 \%)$ were male. Patients in the lowest $25 \%$ of the values obtained as a result of the TPI determined specifically for gender were evaluated sarcopically. With this method, 30 (25\%) patients were considered sarcopenic. The lower quarter for men was $4,23 \pm 1,52 \mathrm{~cm}^{2} / \mathrm{m}^{2}$, while for women it was $2,73 \pm 1,08 \mathrm{~cm}^{2} /$ $\mathrm{m}^{2}$. Thirty-four $(28,3 \%)$ patients were obese and $3(8,4 \%)$ of them were evaluated as sarcopenic obese. PD was performed in $97(80,8)$ patients, while the remaining $23(19,2 \%)$ patients underwent DP or TP. Seven $(5,8 \%)$ patients were operated after neoadjuvant therapy. Stage 2 and 3 patients made up $90,9 \%$ of the study population. 72 (60\%) patients were reported as negative node. No significant difference was found between sarcopenic and non-sarcopenic groups in terms of age, gender, operation, ASA score, TNM stage, and lymph node status.

The relationship between postoperative results and sarcopenia has been summarized in Table 2 . The average operation time was $212,16 \pm 35,25 \mathrm{~min} .45(37,5 \%)$ patients had

\begin{tabular}{|c|c|c|c|c|}
\hline Variables & Total & Sarcopenic $(n=30)$ & Non-sarcopenic $(n=90)$ & $p$-value \\
\hline Age & $58,03 \pm 7,61$ & $59,03 \pm 6,74$ & $57,7 \pm 7,88$ & 0,408 \\
\hline Gender (Male) & $76(63,3)$ & $19(63,3)$ & $57(63,3)$ & 0,590 \\
\hline ASA score & & & & 0,670 \\
\hline 1 & $12(10)$ & $1(3,3)$ & $11(12,2)$ & \\
\hline 2 & $49(40,8)$ & $9(30)$ & $40(44,4)$ & \\
\hline 3 & $59(49,2)$ & $20(66,7)$ & $39(43,4)$ & \\
\hline BMI $\left(\mathrm{kg} / \mathrm{m}^{2}\right)$ & $27,49 \pm 4,41$ & $25,43 \pm 4,77$ & $28,17 \pm 4,08$ & 0,003 \\
\hline Weight loss \% & $6,27 \pm 8,60$ & $8,65 \pm 9,57$ & $5,48 \pm 8,16$ & 0,102 \\
\hline Operation & & & & 0,034 \\
\hline PD & $97(80,8)$ & $28(93,3)$ & $69(76,7)$ & \\
\hline Non-PD & $23(19,2)$ & $2(6,7)$ & $21(23,3)$ & \\
\hline TNM stage & & & & 0.754 \\
\hline 1 & $11(9,1)$ & $2(6,7)$ & $9(10)$ & \\
\hline 2 & $59(49,2)$ & $14(46,7)$ & $45(50)$ & \\
\hline 3 & $50(41,7)$ & $14(46,7)$ & $36(40)$ & \\
\hline Lymph node status & & & & 0,258 \\
\hline No & $72(60)$ & $16(53,3)$ & $56(62,2)$ & \\
\hline N1 & $48(40)$ & $14(46,7)$ & $34(37,8)$ & \\
\hline Neoadjuvant treatment & $7(5,8)$ & $3(10)$ & $4(4,4)$ & 0,239 \\
\hline $\operatorname{TPI}\left(\mathrm{cm}^{2} / \mathrm{m}^{2}\right)$ & $4,68 \pm 1,61$ & $3,06 \pm 0,87$ & $5,22 \pm 1,43$ & $0,001>$ \\
\hline
\end{tabular}

Numerical data were given as mean \pm standard deviation. ASA: American Society of Anesthesiology score, BMI: Body Mass Index, PD: Pancreaticoduodenectomy, TNM: Tumor-Node-Metastasis, TPI: Total Psoas Index. 


\begin{tabular}{|c|c|c|c|c|}
\hline Variables & Total & Sarcopenic $(n=30)$ & Non-sarcopenic $(n=90)$ & $p$-value \\
\hline Operation time (min.) & $212,16 \pm 35,25$ & $220 \pm 31,48$ & $209 \pm 36,20$ & 0,161 \\
\hline Blood loss & & & & 0,045 \\
\hline$>1000 \mathrm{ml}$ & $12(10)$ & $6(20)$ & $6(6,7)$ & \\
\hline $1000 \mathrm{ml}>$ & 108(90) & $24(80)$ & $84(93,3)$ & \\
\hline $\begin{array}{l}\text { Clavien Dindo } 3 \text { and above } \\
\text { complications }\end{array}$ & $45(37,5)$ & $20(66,7)$ & $25(27,8)$ & $0,001>^{*}$ \\
\hline \multicolumn{5}{|l|}{ Pancreatic fistula } \\
\hline Grade A & & & & 0,552 \\
\hline Grade B & $9(7,5)$ & $5(16,7)$ & $4(4,4)$ & \\
\hline \multirow[t]{2}{*}{ Grade C } & $12(10)$ & $4(13,3)$ & $8(8,9)$ & \\
\hline & $0(0)$ & $0(0)$ & $0(0)$ & \\
\hline Cardiac Complications & $20(16,7)$ & $10(33,8)$ & $10(11,1)$ & $0,007^{*}$ \\
\hline Pulmonary Complications & $56(46,7)$ & $21(70)$ & $35(38,9)$ & $0,003^{*}$ \\
\hline Infective Complications & $29(24,2)$ & $4(13,3)$ & $25(27,8)$ & 0,084 \\
\hline Wound Complications & $26(21,7)$ & $4(13,3)$ & $22(24,4)$ & 0,153 \\
\hline Delayed Gastric Emptying & $22(18,3)$ & $5(16,7)$ & $17(18,9)$ & 0,512 \\
\hline Hospital Stay (day) & $11,42 \pm 7,02$ & $14,23 \pm 7,41$ & $10,48 \pm 6,67$ & $0,003^{*}$ \\
\hline Intensive Care Stay (day) & $3,38 \pm 4,40$ & $4,23 \pm 3,71$ & $3,09 \pm 4,59$ & 0,219 \\
\hline $\begin{array}{l}30 \text { day/in-hospital } \\
\text { mortality }\end{array}$ & $8(6,7)$ & $2(6,7)$ & $6(6,7)$ & 0,681 \\
\hline Reoperation & $8(6,7)$ & $1(3,3)$ & $7(7,8)$ & 0,359 \\
\hline
\end{tabular}

Numerical data were given as mean \pm standard deviation.

Clavien-Dindo grade 3 and above complications. Cardiac complications developed in $20(16,7 \%)$ patients, pulmonary in $56(46,7 \%)$ patients, infective in $29(24,2 \%)$ patients, and wound-related complications in $26(21,7 \%)$ patients. Delayed gastric emptying occurred in $22(18,3 \%)$ patients and nasogastric decompression was performed. Based on the clinical condition of the patients and the postoperative drain and blood amylase levels, Grade A pancreatic fistula was observed in 9 $(7,5 \%)$ patients, Grade B fistula in $12(10 \%)$ patients but Grade $\mathrm{C}$ fistula was not observed. The average length of hospital stay was $11,42 \pm 7,02$ days, and the patients needed an average of $3,38 \pm 4,40$ days of intensive care. There was a significant difference between the two groups in terms of length of hospital stay, more in the sarcopenic group $(p=0,003)$. There were 8 $(6,7 \%)$ patients who developed mortality within 1 month after the operation or during hospitalization. The incidence of cardiac and pulmonary complications was significantly different between the groups $(p=0,007, p=0,003)$. When all complications were evaluated, a strong relationship was found between Clavien-Dindo Grade 3 and above complications and sarcopenia $(p=0,001>)$.

Univariate and multivariate analysis of risk factors effective on postoperative serious complications have been summerized in Table 3. When all variables that may affect postoperative serious complications are analyzed, age (OR: 1,08. 95\% CI: 1,01 $\sim 1,15 . p=0,013$ ), ASA score (OR: 2,84. 95\% CI 1,62 4,97. $p=$

\begin{tabular}{|c|c|c|c|c|}
\hline \multirow[b]{2}{*}{ Variable } & \multicolumn{2}{|c|}{ Univariate analysis } & \multicolumn{2}{|c|}{ Multivariate analysis } \\
\hline & OR $(95 \% \mathrm{Cl})$ & $p$-value & $\begin{array}{l}\text { Adjusted OR } \\
\text { (95\% Cl) }\end{array}$ & $p$-value \\
\hline Age & $3.37(0,07 \sim 0,28)$ & 0,001 & $1,08(1,01 \sim 1,15)$ & $0,013^{*}$ \\
\hline ASA score & $4,37(1.96 \sim 9.72)$ & $0,001>$ & $2,84(1,62 \sim 4,97)$ & $0,043^{*}$ \\
\hline TPI & $4,81(2,42 \sim 8,70)$ & $0,001>$ & $3,61(1,58 \sim 5,74)$ & $0,001>$ \\
\hline
\end{tabular}

* OR: Odds-Ratio, Cl: Confidence Interval, ASA: American Society of Anesthesiologist,TPI:Total Psoas Index.

0,043) and TPI (OR: 3,61. 95\% CI: 1,58 5,74. $p=0,001>$ ) have been identified as independent risk factors.

\section{Discussion}

Many factors affect postoperative results in pancreatic cancer, which has a very low survival rate even after curative resection. Serious morbidities may occur after postoperative complications and disease prognosis is affected. While improved surgical technique, increased surgical experience, better anesthesia and intensive care process and improvements in parenteral nutrition make PD a safer procedure, surgery still carries a high risk of postoperative morbidity and mortality. ${ }^{20,21}$ In addition to knowing the treatment and 
management of postoperative complications, it is necessary to predict these complications. This is important in determining the necessary clinical treatment options and appropriate timing in the application of adjuvant therapy. ${ }^{5,6}$ In addition, this prediction may improve patient selection prior to surgical resection. ${ }^{7,8}$

In recent years, sarcopenia has been defined as a syndrome characterized by progressive loss of skeletal muscle mass and can cause consequences such as physical disability, low quality of life, and even death. ${ }^{10-12}$ It also reflects the patient's nutritional status and condition. Like many other factors, sarcopenia has been proven by several randomized studies that affect postoperative results. Some studies have shown that sarcopenia is a negative prognostic factor for esophageal, gastric, ${ }^{22}$ and colorectal cancer. ${ }^{23}$

Several methods for the diagnosis of sarcopenia have been described in the literature. The diagnostic accuracy and sensitivity of the methods based on measurement of muscle mass with radiological imaging compared to anthropometric methods were found to be higher. Based on the fat density values, Hounsfield unit average calculation is one of the methods used in the diagnosis of sarcopenia. TPI is a special measurement based on the ratio of psoas muscle area to the patient's height and reflects the patient's sarcopenia status. According to the international cancer cachexia consensus, TPI value was defined as $<55 \mathrm{~cm}^{2} / \mathrm{m}^{2}$ for men and $<39 \mathrm{~cm}^{2} /$ $\mathrm{m}^{2}$ for women as sarcopenia. ${ }^{24}$ In our study, patients in the lowest $25 \%$ of the TPI value that was evaluated specifically for gender were evaluated as sarcopenic. According to the results obtained in our study, it is seen that TPI value has a significant relationship with postoperative results in pancreatic cancer patients.

TPI values can be easily determined by the patient's preoperative staging CT images and are not a time-consuming procedure. Considering that CT is applied to each patient for preoperative staging, there is no additional radiation exposure and no cost burden. There are also data supporting the use of magnetic resonance imaging (MRI) in staging and operative planning in pancreatic cancer patients. ${ }^{25}$ MRI has previously been used to measure sarcopenia, and can therefore be used preoperatively to perform this function, but CT is considered to be more appropriate for use due to additional cost and possible contraindications of MRI, and therefore we used CT data in our study.

While the focus of our study was the correlation between sarcopenia and surgical outcomes, its relationship with survival has been previously studied. As a result of the analysis of the data, sarcopenia was found as an independent predictor in severe postoperative complications. The most important reason for this may be postoperative limited mobilization due to low muscle strength and the inability to tolerate the negative catabolic state after surgery due to the current cachexia. Also in our study, cardiac and pulmonary complications were more common in the sarcopenic group. The most common extra-abdominal complications in pancreatic surgery are of lung origin and the detection and treatment of sarcopenia in elderly patients with underlying chronic lung disease can contribute to reducing the risk of mortality. In our study, the length of hospital stay was found to be significantly longer in the sarcopenic group. Effective preoperative treatment of sarcopenia is also important in terms of preventing secondary complications originating from hospital and reducing treatment costs.

In the light of these data, the potential of reducing the patient's degree of sarcopenia by interfering with medical and nutritional support can direct the selection of the right patient for the operation and clinical decision-making, especially in the elderly population. Patients who are in the high-risk group in terms of complications may be directed to neoadjuvant chemotherapy during this period by applying concurrent treatments such as nutrition and exercise treatments to reduce the degree of sarcopenia before surgical resection. This approach can lead patients to a better outcome that can ultimately alter patients' prognosis by reducing the postoperative complication rates as well as potential benefits of neodjuvant therapy such as increased surgical curability by reducing tumor size and predicting adjuvant therapy response.

The most important limitation of our study is being a retrospective study performed from a single center and a study with a limited number of samples. It is also possible that performing the operations by different surgeons and possible selection bias would have affected postoperative results. In addition, there is no universally accepted definition of CT-based assessment for sarcopenia in the literature due to differences in physical condition among people. Despite all these limitations, we believe our results are valuable to help understanding the effect of sarcopenia on postoperative outcomes in patients with pancreatic cancer.

As a result, sarcopenia is an important indicator in predicting serious complications after resection in pancreatic cancer. TPI is a patient-specific value that can be easily obtained from preoperative imaging, and given its ease of measurement and its relationship to postoperative complications, it can be widely used to help predict patient morbidity, potentially alter the clinical course of patients by identifying sarcopenic patients, and reduce postoperative complication rates. Determining the degree of sarcopenia can affect patient selection, predictability of possible serious complications, elective operation preparation process with a combination of nutrition and exercise therapy in a particular patient group and decisions regarding adjuvant or neoadjuvant therapy.

\section{Acknowledgment}

I would like to thank all members of the Turkish Surgical Oncology Association supported writing of this article.

\section{Author Contribution}

Mercan U: Data collection and writing

Erşen O: Statistical analysis

Unal AE: Critical review

\section{Disclosure Statement}

There is no conflict of interest in writing of this article. No financial support or funding has been received. 


\section{References}

1. Siegel R, Naishadham D, Jemal A. Cancer statistics, 2012. CA Cancer J Clin 2012;62(1):10-29.

2. Bilimoria KY, Bentrem DJ, Ko CY, Ritchey J, Stewart AK, Winchester DP et al. Validation of the 6th. edition AJCC Pancreatic Cancer Staging System: report from the National Cancer Database. Cancer 2007;110(4):738-44.

3. Winter JM, Brennan MF, Tang LH, D'Angelica MI, DeMatteo RP, Fong Y et al. Survival after resection of pancreatic adenocarcinoma: Results from a single institution over three decades. Ann Surg Oncol. 2012;19(1):16975.

4. Sun RC, Button AM, Smith BJ, Leblond RF, Howe JR, Mezhir JJ A comprehensive assessment of transfusion in elective pancreatectomy: Risk factors and complications. J Gastroint Surg. 2013;17(4);627-35.

5. Van der Gaag NA, Harmsen K, Eshuis WJ, Busch ORC, Van GulikTM, Gouma DJ. Pancreatoduodenectomy associated complications influence cancer recurrence and time interval to death. Eur J Surg Oncol. 2014;40(5):551-8.

6. Valle JW, Palmer D, Jackson R, Cox T, Neoptolemos JP, Ghaneh P et al. Optimal duration and timing of adjuvant chemotherapy after definitive surgery for ductal adenocarcinoma of the pancreas: ongoing lessons from the ESPAC-3 study. J Clin Oncol. 2014;32(6):504-12.

7. Vollmer CM, Sanchez N, Gondek S, McAuliffe J, Kent TS, Christein JD et al. Pancreatic Surgery Mortality Study Group. A root-cause analysis of mortality following major pancreatectomy. J Gastroint Surg. 2012;16(1): 89-103.

8. Kneuertz PJ, Pitt HA, Bilimoria KY, Smiley JP, Cohen ME, Ko CY et al. Risk of morbidity and mortality following hepato-pancreato-biliary surgery. J Gastroint Surg. 2012;16(9):1727-35.

9. Peng P, Hyder O, Firoozmand A, Kneuertz P, Schulick RD, Huang D et al. Impact of sarcopenia on outcomes following resection of pancreatic adenocarcinoma. J Gastroint Surg, 2012;16(8):1478-86.

10. Cruz-Jentoft AJ, Baeyens JP, Bauer JM, Boirie Y, Cederholm T, Landi F et al. Sarcopenia: European consensus on definition and diagnosis: Report of the European Working Group on Sarcopenia in Older People. Age Ageing. 2010;39(4):412-23.

11. Delmonico MJ, Harris TB, Lee JS, Visser M, Nevitt M, Kritchevsky SB et al. Alternative definitions of sarcopenia, lower extremity performance and functional impairment with aging in older men and women. J Am Geriatr Soc. 2007; 55(5):769-74.

12. Goodpaster BH, Park SW, Harris TB, Kritchevsky SB, Nevitt M, Schwartz AV et al. The loss of skeletal muscle strength, mass, and quality in older adults: The health, aging and body composition study. J Gerontol A Biol Sci Med Sci. 2006;61(10):1059-64.
13. Kim TN, Choi KM. Sarcopenia. Definition, epidemiology, and pathophysiology. J Bone Metab. 2013; 21-30.

14. Reisinger KW, van Vugt JL, Tegels JJ, Snijders C, Hulsewé KW, Hoofwijk AG et al. Functional compromise reflected by sarcopenia, frailty, and nutritional depletion predicts adverse postoperative outcome after colorectal cancer surgery. Ann Surg, 2015;261(2):345-52.

15. Ongaro E, Buoro V, Cinausero M, Caccialanza R, Turri A, Fanotto V et al. Sarcopenia in gastric cancer: When the loss costs too much. Gastric Cancer. 2017;20(4):563-72

16. Shen Y, Hao Q, Zhou J, Dong B. The impact of frailty and sarcopenia on postoperative outcomes in older patients undergoing gastrectomy surgery: A systematic review and meta-analysis. BMC Geriatr., 2017;17(1):188

17. Japan Pancreas Society (2009) General rules for the study of pancreatic cancer, 6th. edn. Kanehara, Tokyo.

18. Dindo D, Demartines N, Clavien PA Classification of surgical complications: A new proposal with evaluation in a cohort of 6336 patients and results of a survey. Ann Surg, 2004;240:205-13.

19. Dusch N, Lietzmann A, Barthels F, Niedergethmann M, Rückert F, Wilhelm TJ. International study group of pancreatic surgery definitions for postpancreatectomy complications: Applicability at a high-volume center. Scand J Surg. 2017:106(3):216-23.

20. Uzunoglu FG, Reeh M, Vettorazzi E, Ruschke T, Hannah P, Nentwich MF et al. Preoperative pancreatic resection (PREPARE) score: A prospective multicenter-based morbidity risk score. Ann Surg. 2014;260:857-63.

21. Winter JM, Cameron JL, Campbell KA, Arnold MA, Chang DC, Coleman J et al. 1423 pancreaticoduodenectomies for pancreatic cancer: A singleinstitution experience. J Gastrointest Surg. 2006;10:1199-210.

22. Fukuda Y, Yamamoto K, Hirao M, Nishikawa K, Nagatsuma Y, Nakayama T et al. Sarcopenia is associated with severe postoperative complications in elderly gastric cancer patients undergoing gastrectomy. Gastric Cancer. 2016;19(3):986-93.

23. Miyamoto Y, Baba Y, Sakamoto Y, Ohuchi M, Tokunaga R, Kurashige J et al. Sarcopenia is a negative prognostic factor after curative resection of colorectal cancer. Ann Surg Oncol. 2015;22(8):2663-68.

24. Fearon K, Strasser F, Anker SD, Bosaeus I, Bruera E, Fainsinger RL et al. Definition and classification of cancer cachexia: An international consensus. The Lancet Oncol., 2011;12(5):489-95.

25. Grimm A, Meyer H, Nickel MD, Nittka M, Raithel E, Chaudry $O$ et al. Repeatability of Dixon magnetic resonance imaging and magnetic resonance spectroscopy for quantitative muscle fat assessments in the thigh. J Cachexia Sarcopenia Muscle., 2018;9(6):1093-100.

This work is licensed under a Creative Commons Attribution-NonCommercial 3.0 Unported License which allows users to read, copy, distribute and make derivative works for non-commercial purposes from the material, as long as the author of the original work is cited properly. 